\title{
Mecanismos de ingresso de dentistas no SUS: uma agenda prioritária para o fortalecimento do Brasil Sorridente
}

\author{
Admission of dentist in Brazilian Universal Health System (SUS): \\ a priority agenda for the strengthening of Smiling Brazil
}

\author{
Mariana Gabriel (https://orcid.org/0000-0001-8824-5827) ${ }^{1}$ \\ Maristela Honório Cayetano (https://orcid.org/0000-0002-0694-4171) ${ }^{1}$ \\ Mariana Murai Chagas (https://orcid.org/0000-0002-9483-6620) ${ }^{1}$ \\ Maria Ercilia de Araujo (https://orcid.org/0000-0003-2689-2556) ${ }^{1}$ \\ Gilles Dussault (https://orcid.org/0000-0002-5976-3454) ${ }^{2}$ \\ Gilberto Alfredo Pucca Junior (https://orcid.org/0000-0002-8781-9857) ${ }^{3}$ \\ Fernanda Campos Sousa de Almeida (https://orcid.org/0000-0003-3745-2759) ${ }^{1}$
}

${ }^{1}$ Departamento de

Odontologia Social,

Faculdade de Odontologia

da Universidade de São

Paulo. Av. Professor Lineu

Prestes 2227, Cidade

Universitária. 05508-000

São Paulo SP Brasil.

marianaodonto@usp.br

${ }^{2}$ Instituto de Higiene

e Medicina Tropical,

Universidade Nova de

Lisboa. Lisboa, Portugal.

${ }^{3}$ Departamento de

Odontologia, Universidade

de Brasília. Brasília DF

Brasil.

\begin{abstract}
This article aims at: i) describing and analyzing the expansion of dental care in the Unified Health System (SUS); ii) Identifying and analyzing the characteristics of hiring dentists' in the public service; iii) characterizing public vacancies, their duties and remuneration. In this descriptive case study, databases of the Ministry of Health were consulted and public tender notices. The findings indicate that $48 \%$ of the dentists enrolled in the National Registry of Health Establishments (CNES) perform care in the SUS, in 13 years there was an increase of $118 \%$ of the municipalities with oral health teams (eSB) implanted. The population coverage estimated by eSB increased by $10.46 \%$ between the years 2007 and 2015. The main mechanism for joining the Dental Specialties Centers (CEO) was the public tender. Primary care salaries ranged from 1.05 to 12.67 Brazilian minimum wages, to 40-hour weekly jobs, and to CEOs from 3.35 to 7.05. It is concluded that, among other measures, the planning of HRH strategies is necessary. The continuity of successes regulatory measures of labor contracts and support to local managers enter the agenda of priority actions of oral health policy.
\end{abstract}

Key words Human Resources in Health, Public Policies, Oral Health
Resumo Este artigo objetiva i) descrever e analisar a expansão do provimento de dentistas no Sistema Único de Saúde (SUS); ii) identificar e analisar as características do vinculo trabalhista dos dentistas com o serviço; iii) caracterizar as vagas em concurso público, no que se refere aos requisitos, atribuições e remuneração. Neste estudo de caso, descritivo, foram consultados bancos de dados do Ministério da Saúde e editais de concurso público. Os achados apontam que 48\% dos dentistas cadastrados no Cadastro Nacional dos Estabelecimentos de Saúde (CNES) realizam atendimento no SUS, em 13 anos observou-se um aumento de $118 \%$ dos municípios com equipes de saúde bucal (eSB) implantadas. A cobertura populacional estimada pelas eSB aumentou 10,46\% entre os anos de 2007 e 2015. O principal mecanismo de ingresso nos Centros de Especialidades Odontológicas (CEO) foi o concurso público. O salário na atenção primária variou de 1,05 a 12,67 salários minimos, para cargos de 40 horas semanais e nos CEOs de 3,35 a 7,05. Conclui-se que é necessário, entre outras medidas, o planejamento de estratégias voltadas aos recursos humanos em saúde. A continuidade dos êxitos alcançados demanda que medidas regulatórias dos contratos de trabalho e apoio aos gestores entrem na agenda das ações da politica em saúde bucal.

Palavras-chave Recursos Humanos em Saúde, Políticas Públicas, Saúde Bucal 


\section{Introdução}

A má distribuição geográfica dos profissionais de saúde é um problema global que afeta inúmeros países $^{1}$, entre eles o Brasil ${ }^{2}$, e corrigir essas distorções certamente contribuirá para melhorar a cobertura dos serviços de saúde. Estudiosos sobre o tema apontam que para alcançar os objetivos de saúde para todos é necessário uma força de trabalho em número suficiente, com profissionais de diferentes áreas competentes, motivados e alocados adequadamente nas diversas regiões geográficas. Além disso, destaca-se a importância de políticas explícitas voltadas para o desenvolvimento dos Recursos Humanos para a Saúde (RHS) $)^{3}$.

Agências internacionais de saúde já fomentam essas discussões há algum tempo e, em 2013, o Brasil sediou a 3a edição do Fórum Global de Recursos Humanos em Saúde, organizado pela Organização Mundial de Saúde (OMS), Organização Pan-Americana da Saúde (OPAS) e Health Alliance Global Workforce. O fórum resultou em um relatório que reforçou a necessidade do compromisso político para a valorização dos RHS nas agendas de saúde pós-2015. Esse relatório motivou a realização desse estudo ao indicar que o governo deve elaborar mecanismos reguladores para assegurar a capacidade de resposta do sistema de saúde ${ }^{4}$. Esse tema também foi destaque no $4^{\circ}$ Fórum Global sobre Recursos Humanos em Saúde realizado em 2017 na cidade de Dublin, Irlanda ${ }^{5}$.

No Brasil, o programa "Mais Médicos" foi responsável por trazer para o centro das discussões políticas o problema da escassez de profissionais de saúde em algumas regiões do país, já que os profissionais tendem a concentrar-se nos grandes centros ${ }^{6}$. O Sistema Único de Saúde (SUS) impõe que o cuidado em saúde seja oferecido de forma integral, e para tanto faz-se necessária a articulação dos serviços com vistas a responder as demandas de promoção, prevenção, cura e reabilitação da população.

Dentre os determinantes que influenciam as decisões dos profissionais de saúde em relação ao local de atuação, destacam-se: pessoais, profissionais, organizacionais, econômicos, políticos e culturais ${ }^{7}$. Sendo assim, os incentivos financeiros não podem ser vistos como a única forma de motivar o recrutamento e retenção dos profissionais ${ }^{8}$, intervenções desenvolvidas em diferentes setores, tais como educação, saúde e emprego, têm apresentado maior impacto em relação a intervenções estanques ${ }^{9}$. Nessa perspectiva a Se- cretaria da Gestão do Trabalho e da Educação na Saúde (SGTES), instituiu a política de Gestão do Trabalho em Saúde, que valoriza o trabalhador para a efetividade e eficiência do SUS garantindo os requisitos básicos para o serviço, entre eles, estão: Plano de Carreira, Cargos e Salários e o vínculo de trabalho com proteção social ${ }^{10}$.

No SUS as formas de contratação de recursos humanos sofreram algumas modificações ao longo dos anos. A Constituição Federal de 1988, instituiu o Regime Jurídico Único (RJU), ou seja, o vínculo estatutário, no qual a forma de ingresso seria unicamente por meio de concurso público. No entanto, em 1998, a emenda constitucional ampliou as formas de vínculos trabalhistas. Atualmente, os contratos também podem ser de acordo com a Consolidação das Leis do Trabalho (CLT) além de permitir o intermédio de organizações para terceirizar a contratação de RHS (Pública ou Privada $)^{11}$.

Neste contexto, por reconhecer a Saúde Bucal como parte integrante e essencial para a saúde geral e para a qualidade de vida da populaçãa ${ }^{12}$, em 2004 o Ministério da Saúde implantou a Política Nacional de Saúde Bucal (PNSB), denominada "Brasil Sorridente", que além de fortalecer a atenção primária, por meio das Equipes de Saúde Bucal (eSB), vinculadas à Estratégia Saúde da Família (ESF), constituiu uma rede de atenção à saúde bucal que foi responsável pela expansão no território nacional dos postos de trabalho de dentistas no SUS. No entanto, verifica-se que, assim como na medicina, a odontologia também apresenta vazios sanitários, resultantes da má distribuição dos dentistas ${ }^{13}$. Considerando essa realidade imposta no Brasil e o quadro conceitual existente sobre os fatores que influenciam a distribuição dos $\mathrm{RHS}^{14}$, este artigo tem como objetivos i) descrever e analisar a expansão do provimento de dentistas no SUS; ii) identificar e analisar as características do vínculo trabalhista dos dentistas com o serviço; iii) caracterizar as vagas em concurso público, no que se refere aos requisitos, atribuições e remuneração.

\section{Metodologia}

Este é um estudo de caso brasileiro, descritivo, que integra um conjunto de pesquisas sobre regulação de RHS desenvolvido pelo Observatório de Recursos Humanos em Odontologia (OBSERVARHODONTO) do Brasil e o Instituto de Higiene e Medicina Tropical (IHMT) da Universidade Nova Lisboa, Lisboa, Portugal. 
Foram utilizadas diferentes fontes de informações, a fim de gerar os dados necessários para o estudo. Utilizou-se dados secundários extraídos de diferentes bancos do Ministério da Saúde, sendo: 1) Departamento de Atenção Básica $(D A B)^{15}$ - para identificar a expansão do provimento de dentistas no SUS (por meio do histórico de implantação das eSB); 2) DATASUS - Tecnologia da Informação a Serviço do SUS e do Cadastro Nacional de Estabelecimentos de Atenção à Saúde $(C N E S)^{16}$ - para identificar o número de dentistas com vínculo no SUS; 3 ) e-gestor Atenção Básica $^{17}$ - para identificar a cobertura populacional das eSB; 4) Programa de Melhoria do Acesso e da Qualidade dos Centros de Especialidades Odontológicas (PMAQ-CEO) - para analisar o vínculo trabalhista dos dentistas com o serviço e a oferta do serviço especializado no Brasil Sorridente nas cinco regiões do país. Nessa fase, utilizou dados provenientes da avaliação externa do PMAQCEO $1^{\circ}$ Ciclo, realizada em $2014^{18}$.

A avaliação externa do PMAQ-CEO foi dividida em três módulos: módulo de observação direta, de entrevista com o gerente e com um profissional da unidade e o último, de satisfação do usuário. Nesse estudo, foram selecionadas perguntas do módulo de entrevista com o gerente e com um profissional da unidade, pois permitiam identificar o número de CEOs no país, a distribuição geográfica dos serviços e o vínculo trabalhista dos dentistas com o serviço, o que determinou a escolha desse componente da rede Brasil Sorridente para esse estudo. Entre os indicadores, os seguintes foram considerados: agente contratante (pergunta VIII.4.1), tipo de vínculo (pergunta VIII.4.2), mecanismo de ingresso (pergunta VIII.4.3.1) e plano de carreira/prêmio financeiro por desempenho (pergunta VIII.5).

Por fim, foi realizada uma pesquisa documental em editais de concursos públicos. O banco de editais foi disponibilizado por uma empresa que anuncia concursos em websites e continha editais de diferentes vagas abertas no país no período de 2006 a 2015. Foram selecionados apenas os editais que contemplavam vagas para dentista com cargos em prefeituras dos municípios brasileiros, o que gerou um total de 210 editais. Esses dados foram organizados no programa SPSS (versão 15.0, SPSS Inc, Chicago, IL) e um sorteio por reposição de amostra selecionou cinco editais por estado (amostra de conveniência) de modo a contemplar as cinco regiões brasileiras (Centro-Oeste, Nordeste, Norte, Sudeste e Sul), Vale ressaltar que em alguns estados o número máximo de editais encontrados foram cinco, sendo assim padronizou-se esse número também para os demais estados, ficando portanto, um total de 135 editais analisados em uma amostra de conveniência. Os editais foram lidos na íntegra e as informações relevantes para o estudo, tais como, aspectos gerais (ano da realização do concurso e região geográfica), e aspectos do cargo (especialidade exigida, nível de atenção, número de vagas ofertadas, cadastro de reserva, vínculo trabalhista, agente contratante, horas trabalhadas e salário), foram analisados aplicando-se estatística descritiva no software SPSS (versão 15.0, SPSS Inc, Chicago, IL).

\section{Resultados}

Os achados serão apresentados em três seções: 1) Expansão do provimento de dentistas no SUS; 2) Características dos vínculos trabalhistas entre dentistas e os serviços; 3) Caracterização dos editais de concursos públicos para dentistas no SUS.

\section{Expansão do provimento de dentistas no SUS}

Cerca de $48 \%$ dos dentistas cadastrados no CNES do Brasil realizam atendimento no SUS (Tabela 1). As regiões do Norte e do Nordeste apresentam a maioria dos dentistas cadastrados no CNES com vínculo no SUS, $61 \%$ e $67 \%$ respectivamente, enquanto que as regiões Centro-Oeste, Sudeste e Sul prevalecem no setor privado, esses dados mostram que o mercado de trabalho odontológico vem se modificando e deixando de ser caracterizado apenas em um setor, mas sim em um mix público-privado ${ }^{19}$.

A implementação do Brasil Sorridente pode ter sido um impulsionador importante desse número, uma vez que em apenas 13 anos observouse um aumento de $118 \%$ dos municípios com eSB implantadas. No fim de 2015 , cerca de $90 \%$ dos municípios brasileiros apresentaram eSB, sendo 22.227 eSB na modalidade 1 (dentista e auxiliar de saúde bucal) e 2.240 eSB na modalidade 2 (dentista, auxiliar de saúde bucal e técnico em saúde bucal). Em relação a cobertura populacional estimada pelas eSB, observou-se aumento de 10,46\% entre os anos de 2007 e 2015 (Tabela 2).

\section{Vínculo trabalhista dos dentistas com o serviço}

Os CEO são referência à atenção básica em saúde, e também articuladores da atenção terci- 
Tabela 1. Número de dentistas cadastrados no CNES

\begin{tabular}{lccc}
\hline \multirow{2}{*}{ Região } & \multicolumn{3}{c}{ Dentistas cadastrados no CNES } \\
\cline { 2 - 4 } & Total & Atende no SUS & Não atende no SUS \\
\hline Centro-Oeste & 10.790 & $4.556(42 \%)$ & $6.234(58 \%)$ \\
Nordeste & 24.785 & $16.490(67 \%)$ & $8.295(33 \%)$ \\
Norte & 6.454 & $3.930(61 \%)$ & $2.524(39 \%)$ \\
Sudeste & 58.231 & $24.823(43 \%)$ & $33.408(57 \%)$ \\
Sul & 21.358 & $8.441(40 \%)$ & $12.917(60 \%)$ \\
Total & 121.618 & $58.240(48 \%)$ & $63.378(52 \%)$ \\
\hline
\end{tabular}

Fonte: Elaboração própria com dados disponíveis do Ministério da Saúde, Banco de dados do Sistema Único de Saúde DATASUS, Cadastro Nacional de Estabelecimentos de Atenção à Saúde - CNES Recursos Humanos, $2015^{16}$.

Tabela 2. Histórico de implantação das Equipes de Saúde Bucal na atenção primária nos municípios brasileiros e cobertura populacional estimada.

\begin{tabular}{lcccc}
\hline Ano & $\begin{array}{c}\text { No de municípios } \\
\text { com eSB }\end{array}$ & $\begin{array}{c}\text { eSB. Modalidade } \\
\text { I }\end{array}$ & $\begin{array}{c}\text { eSB. Modalidade } \\
\text { II }\end{array}$ & $\begin{array}{c}\text { Cobertura populacional } \\
\text { estimada eSB }\end{array}$ \\
\hline 2002 & 2.302 & 3.819 & 442 & $*$ \\
2003 & 2.787 & 5.631 & 539 & $*$ \\
2004 & 3.184 & 8.234 & 717 & $\star$ \\
2005 & 3.897 & 11.717 & 886 & $\star$ \\
2006 & 4.285 & 14.019 & 1.067 & $*$ \\
2007 & 4.294 & 14.563 & 1.131 & $29.90 \%$ \\
2008 & 4.597 & 16.423 & 1.384 & $33.29 \%$ \\
2009 & 4.717 & 17.465 & 1.517 & $34.61 \%$ \\
2010 & 4.830 & 18.731 & 1.693 & $36.54 \%$ \\
2011 & 4.883 & 19.492 & 1.933 & $38.35 \%$ \\
2012 & 4.901 & 20.155 & 2.048 & $38.97 \%$ \\
2013 & 4.971 & 21.016 & 2.134 & $39.46 \%$ \\
2014 & 5.018 & 22.066 & 2.257 & $39.90 \%$ \\
2015 & 5.014 & 22.227 & 2.240 & $40.36 \%$ \\
\hline
\end{tabular}

${ }^{*}$ Dados não disponíveis para o período.

Fonte: Elaboração própria com dados do Departamento de Atenção Básica e do e-gestor Atenção Básica ${ }^{15,17}$.

ária em saúde bucal no SUS. O Brasil Sorridente conta com 1.034 CEOs, sendo que 930 participaram do PMAQ-CEO (62 no Centro-Oeste, 355 no Nordeste, 59 no Norte, 337 no Sudeste e 117 no Sul). A implantação dos CEOs também foi responsável pela expansão dos postos de trabalho para dentista no SUS e os dados revelam que o principal mecanismo de ingresso no CEO foi o concurso público, porém cerca de 33\% não foi por essa via (Tabela 3 ).

Segundo dados do PMAQ CEO, em relação ao agente contratante, observa-se que $20 \%$ dos dentistas analisados não são contratados a partir da administração direta, mas sim por meio de outras formas de contratação: consórcio in- termunicipal de direito público, consórcio intermunicipal de direito privado, fundação pública de direito público, fundação pública de direito privado, organização social (OS), organização da sociedade civil de interesse público (OSCIP), entidade filantrópica, empresa, cooperativa e outros que não foram tipificados (Tabela 3 ).

Em relação a oportunidade de crescimento profissional, no total de CEOs, 307 (33\%) apresentavam contratos com plano de carreira para os dentistas. No entanto, existiu variações em relação as regiões geográficas, sendo 50\% dos CEOs do Centro-Oeste, $15 \%$ do Nordeste, $41 \%$ do Norte, $40 \%$ do Sudeste e $54 \%$ da região Sul (Tabela 3). 


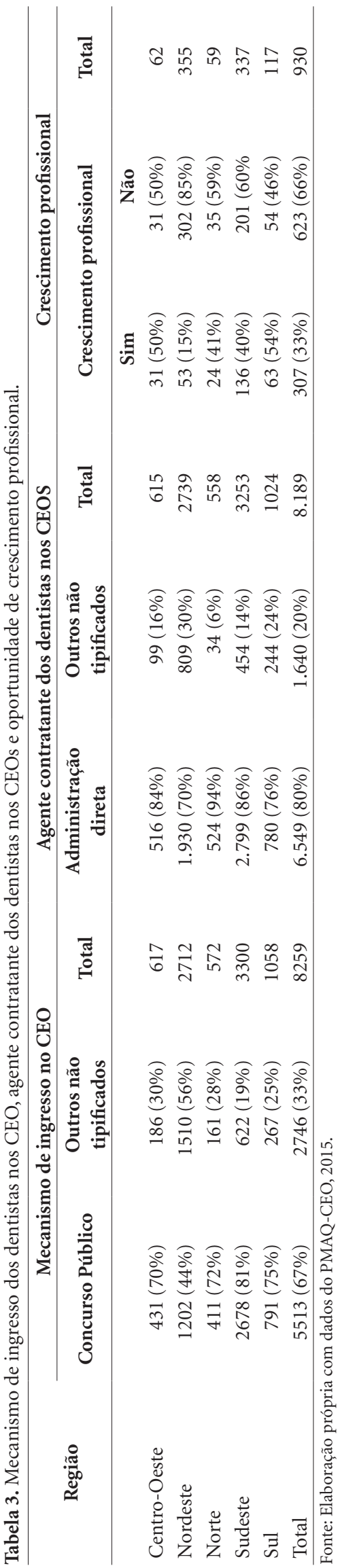

\section{Caracterização dos editais de concursos públicos para dentistas no SUS}

\section{Requisitos e atribuições}

Um total de 715 vagas, destinadas a dentistas, para compor o quadro das prefeituras de municípios brasileiros, das cinco regiões geográficas, foram analisadas em uma amostra de conveniência de 135 editais ( 5 por estado). Essas vagas previam a atuação em diferentes pontos da rede de atenção no SUS (Tabela 4), no entanto, no nível de atenção primária, a maioria dos editais, não especificava se a vaga era para compor as equipes da Estratégia Saúde da Família (ESF). Vagas destinadas a cadastro de reserva foram identificadas em 20 editais, no entanto destes apenas 7 não contemplavam nenhuma vaga para atuação imediata.

Em relação as exigências para ocupação da vaga todas exigiam curso superior de graduação em odontologia e 102 (14\%) das vagas exigiam curso de especialização para a ocupação dos cargos. É importante destacar que embora os CEO realizem atendimentos especializados não é requisito ser dentista especialista.

Nesse estudo buscou-se caracterizar algumas condições relevantes de contratação nos editais abertos nos diferentes municípios do país. Verificou-se que a maioria dos editais (45\%) eram de prefeituras de municípios com populações entre 10.000 a 50.000 mil habitantes, 35\% com população com até 10.000 e $20 \%$ com população maior que 50.000. Em relação ao Índice de Desenvolvimento Humano Municipal (IDHM) 98\% dos editais pertenciam a municípios com IDHM considerado médio pelo Programa das Nações Unidas para o Desenvolvimento (PNUD Brasil), ou seja, entre $(0,500$ a 0,799$)$.

\section{Remuneração}

Os salários oferecidos nos editais foram organizados por região de acordo com o nível de atenção e a carga horária de trabalho da vaga (Tabela 5). A carga horária dos editais variou entre 10 a 40 horas/semanais, mas a grande maioria exigia 20 ou 40 horas/semanais. Como as referências salarias eram de um período de dez anos, fixamos a nossa análise a partir da razão do salário do edital com o salário mínimo brasileiro referente ao ano do concurso ${ }^{20}$.

\section{Discussão}

Verifica-se que em pouco mais de uma década, houve um aumento expressivo da disponibili- 
Tabela 4. Vagas oferecidas nos editais analisados de acordo com as cinco regiões brasileiras.

\begin{tabular}{lrrrrr}
\hline \multirow{2}{*}{ Região } & \multicolumn{1}{c}{ Editais } & \multicolumn{1}{c}{ Vagas } & \multicolumn{2}{c}{ Vagas por nível de atenção - n (\%) } \\
\cline { 2 - 6 } & & & Atenção primária & CEO & Não Informada \\
\hline Centro-Oeste & 15 & $55(8 \%)$ & $8(15 \%)$ & - & $47(85 \%)$ \\
Nordeste & 45 & $201(28 \%)$ & $87(43 \%)$ & $45(22 \%)$ & $69(34 \%)$ \\
Norte & 35 & $186(26 \%)$ & $6(3 \%)$ & - & $180(97 \%)$ \\
Sudeste & 20 & $251(35 \%)$ & $139(55 \%)$ & $1(0,3 \%)$ & $111(44 \%)$ \\
Sul & 15 & $22(3 \%)$ & $7(32 \%)$ & $5(23 \%)$ & $10(45 \%)$ \\
Total & 130 & $715(100 \%)$ & $247(35 \%)$ & $51(7 \%)$ & $417(58 \%)$ \\
\hline Fonte: Elaboraccão própria com dados dos editais analisados.
\end{tabular}

Tabela 5. Remuneração referente as características das vagas e a região*.

\begin{tabular}{|c|c|c|c|c|c|c|}
\hline \multirow{4}{*}{ Região } & \multicolumn{6}{|c|}{ Nível de Atenção } \\
\hline & \multicolumn{2}{|c|}{ Atenção Primária } & \multicolumn{2}{|c|}{ CEO } & \multicolumn{2}{|c|}{ Não Informado } \\
\hline & \multicolumn{6}{|c|}{ Carga Horária } \\
\hline & $20 \mathrm{~h} / \mathrm{s}$ & $40 \mathrm{~h} / \mathrm{s}$ & $20 \mathrm{~h} / \mathrm{s}$ & $40 \mathrm{~h} / \mathrm{s}$ & $20 \mathrm{~h} / \mathrm{s}$ & $40 \mathrm{~h} / \mathrm{s}$ \\
\hline \multicolumn{7}{|l|}{ Centro-Oeste } \\
\hline Mínimo & 7,11 & 4,79 & - & - & 1,67 & 3,29 \\
\hline Máximo & 7,11 & 5,78 & - & - & 5,40 & 8,56 \\
\hline \multicolumn{7}{|l|}{ Nordeste } \\
\hline Mínimo & - & 1,05 & 2,73 & - & 1,72 & 1,44 \\
\hline Máximo & - & 8,95 & 2,73 & - & 2,23 & 6,45 \\
\hline \multicolumn{7}{|l|}{ Norte } \\
\hline Mínimo & - & 4,13 & - & - & 2,86 & 2,00 \\
\hline Máximo & - & 7,06 & - & - & 3,42 & 6,45 \\
\hline \multicolumn{7}{|l|}{ Sudeste } \\
\hline Mínimo & 2,87 & 2,86 & 3,38 & 3,35 & 1,43 & 3,58 \\
\hline Máximo & 2,87 & 12,67 & 3,38 & 3,35 & 4,10 & 4,45 \\
\hline \multicolumn{7}{|l|}{ Sul } \\
\hline Mínimo & 2,41 & 3,79 & 4,17 & 7,05 & 4,31 & 3,22 \\
\hline Máximo & 3,37 & 5,63 & 4,17 & 7,05 & 4,84 & 11,51 \\
\hline
\end{tabular}

dade de postos de trabalho e que aproximadamente metade da força de trabalho ocupada em odontologia apresenta vínculo com o SUS. O aumento das eSB pode ser observado não apenas nos grandes centros, mas em todo o país, o que certamente vem colaborando para corrigir parte das iniquidades e reduzir, em alguma medida, as desigualdades na utilização dos serviços ${ }^{21}$. No entanto, a cobertura populacional ainda é insuficiente e coloca em risco o princípio da universalidade da saúde, conforme preconizado pelo SUS. Quanto ao vínculo trabalhista nos CEOs, identificou-se que $20 \%$ dos dentistas são contratados por modalidades de gerência que não são subor- dinadas à administração direta do Estado. Esse tema é bastante polêmico entre principais atores políticos do SUS, existindo opiniões contrárias e favoráveis $^{22}$. Girardi e Carvalho ${ }^{23}$, apontam que identificar os agentes contratantes é fundamental para desenhar estratégias e instrumentos de gestão voltados aos $\mathrm{RHS}^{23}$. Existem evidências que diferenças nos vínculos trabalhistas entre os profissionais de um mesmo serviço, podem apresentar consequências negativas sobre o processo de trabalho da equipe ${ }^{24}$.

Nas análises dos editais para concursos públicos, verificou-se que embora a contratação via concurso proporcione estabilidade ao profissio- 
nal, por não estar atrelado a possíveis mudanças políticas e garantir os direitos trabalhistas, os baixos salários encontrados em alguns editais, as variações de remuneração e de jornada de trabalho para desempenhar o mesmo cargo podem desestimular o profissional e comprometer as iniciativas do programa de desprecarização do trabalho no SUS $^{25}$. A literatura aponta que para que seja alcançada a cobertura universal em saúde é necessário disponibilidade, acessibilidade, aceitabilidade e qualidade da força de trabalho em saú$\mathrm{de}^{2}$. Com isso, faz-se igualmente necessário que além da disponibilidade dos profissionais, exista monitoramento e avaliação de todo o conjunto de intervenções que estão sendo feitos no setor saúde, de modo a identificar os resultados da estratégia implementada e possibilitar a investigação dos fatores contextuais que influenciam seu sucesso ou fracasso ${ }^{26}$.

Nesse estudo, buscou-se abordar a expansão dos dentistas no SUS e analisá-las sob a ótica das relações de trabalho, por considerar que a estabilidade profissional é fundamental para motivar e melhorar o desempenho dos profissionais no setor saúde ${ }^{27}$. Com a descentralização da gestão, estabelecida a partir da Constituição Federal de 1988 e regulamentada pelas Leis 8.080/90 (Lei Orgânica da Saúde) e 8.142/9028 o município se tornou o grande empregador dos profissionais de saúde, inclusive dos dentistas ${ }^{29}$. Atualmente, o país apresenta 5.570 municípios, sendo importante destacar que cada um apresenta realidades socioeconômicas diversas e muitas vezes precárias, colocando o Brasil entre os países que apresentam maior taxa de desigualdade no mundo ${ }^{30}$. Essa realidade brasileira, marcada pelas diversidades regionais, impõe importantes nuances para serem analisadas, entre elas, Campos et al. ${ }^{31}$ questionam: Qual a contrapartida que o SUS oferece para com os municípios em relação a estrutura e infraestrutura, instrumentos e modelos de gestão pública para garantir uma missão constitucional? No caso do Brasil Sorridente as diretrizes da política apontam a necessidade de: "assumir o compromisso de qualificação da atenção bási$\mathrm{ca}$, garantindo qualidade e resolutividade, independentemente da estratégia adotada pelo município para sua organização" ${ }^{32}$ (p.4). Se por um lado a política orienta que as eSB proporcionem a qualidade no cuidado, por outro lado pode falhar em não apresentar os mecanismos organizacionais necessários para fazê-lo. A Política deve ter um papel indutor na qualificação do trabalho, atualmente para credenciar uma eSB, implantar um CEO ou qualquer outro componente da rede Brasil Sorridente é necessário que o gestor municipal apresente uma proposta ao Conselho Municipal de Saúde e, se aprovada, deve encaminhar à Comissão Intergestores Bipartite (CIB) do Estado. No caso do credenciamento das eSB, essa proposta deve incluir, entre outras informações, a descrição da forma de recrutamento, seleção e contratação dos profissionais da equipe. Caso a proposta seja aprovada, o município recebe o repasse do recurso para implantação do componente solicitado ${ }^{33}$, entretanto, não há um monitoramento do processo. É notável a importância de algumas iniciativas do Ministério da Saúde em relação aos vínculos de trabalho, como por exemplo, a Política de Carreiras para o SUS e a Mesa de Negociações, mas é necessário também que as práticas de contratação de RHS entrem na agenda da política do Brasil Sorridente, pois além de ser considerada uma ferramenta poderosa para uma gestão mais eficaz, pode ser fundamental para melhorar a qualidade das medidas de provimento de $\mathrm{RHS}^{34}$. Reconhecendo a evidência, que os incentivos considerados eficazes para os trabalhadores da saúde devem apresentar: objetivos claros; ser realista; refletir as necessidades e as preferências dos profissionais de saúde; ser bem desenhado e estrategicamente apto para suas finalidades; ser adequados para o contexto local; ser justo; equitativo e transparente; ser mensurado e acompanhado; incorporar elementos financeiros e não financeiros ${ }^{35}$. Fica claro que apenas ampliar o número de profissionais de saúde e não estabelecer um quadro regulatório nacional de RHS, que contemple essas evidências, pode comprometer a sustentabilidade das políticas e agravar o acesso à saúde, principalmente de comunidades remotas e vulneráveis.

Embora os postos de trabalho no SUS tenham aumentado, pesquisas ainda apontam uma tendência crescente na má distribuição dos dentistas no país, principalmente por considerarem que as concentrações das instituições formadoras e o melhor poder aquisitivo da população nos grandes centros influenciam na decisão do lugar de atuação dos dentistas, já que esta classe de profissional busca uma dupla prática público/priva$\mathrm{da}^{36}$. Esse é um tema crucial, porém ainda pouco explorado nos sistemas de saúde ${ }^{37}$. A falta de um plano de cargos e salários pode colaborar com esse comportamento e favorecer a rotatividade dos profissionais no SUS ${ }^{38}$. O Brasil Sorridente organiza a rede de cuidados de saúde bucal com base nos preceitos da ESF, que preconiza uma jornada de trabalho de 40 horas/semanais. Essa estratégia reconhece e valoriza a necessidade do vínculo tra- 
balhista e a distribuição dos profissionais, além do compromisso e da parceria entre os profissionais e a comunidade ${ }^{39}$, no entanto, algumas incoerências são relatadas, como por exemplo, flexibilidade da jornada de trabalho em detrimento de formas precárias de contratação $0^{40}$. Os resultados apresentados apontam, que essa pode ser uma realidade também na odontologia, uma vez que se identificou editais de concurso público destinados às eSB com jornada de trabalho de 20 horas/semanais. Essa realidade pode colaborar para o distanciamento do profissional com o serviço e para possíveis conflitos éticos relacionados ao mercado público/privado, como por exemplo, a conquista do status e do ganho financeiro no serviço privado e a garantia de estabilidade e vantagens trabalhistas no serviço público ${ }^{19}$. A valorização do profissional deve ser fortalecida, para isso, Nogueira ${ }^{41}$ aponta para a importância de uma situação de legalidade dos vínculos por meio de servidores devidamente concursados. Um estudo realizado em uma macrorregião do estado de Minas Gerais verificou os critérios de seleção utilizados para contratação dos trabalhadores de saúde e identificou que os secretários municipais utilizavam diversos critérios de seleção e que apenas $20 \%$ deles eram por meio de concursos públicos. O estudo também chama a atenção para algumas formas de seleção adotadas pelos secretários, como por exemplo, a indicação política, que segundo o autor são: "alternativas de incorporação arcaicas do empreguismo, clientelismo e patrimonialismo" ${ }^{42}$ (p.925).

Este estudo apresenta limitações inerentes ao método, pois baseia-se em dados secundários. Essas fontes podem apresentar vieses uma vez que dependem da atualização constante dos órgãos responsáveis pelas informações. Para diminuir esse viés foram utilizadas várias fontes de dados. Além disso, o OBSERVARHODONTO está desenvolvendo pesquisas qualitativas para aprofundar essas análises.

Em conclusão, entende-se que reconhecer os facilitadores e as barreiras contextuais relacionadas a distribuição dos profissionais da saúde se torna essencial para planejar as ações futuras. Quanto aos facilitadores, pode-se destacar as inúmeras políticas voltadas aos RHS em diferentes setores no Brasil ${ }^{43}$, políticas específicas sobre a problemática, tais como o PROVAB e o Mais Médicos, bem como outras que embora não apresentassem apenas esse objetivo proporcionaram a expansão da oferta de serviços no território nacional, como por exemplo o Brasil Sorridente.

Quanto às barreiras, esse estudo contribuiu para identificar fragilidades existentes nas rela- ções de trabalho dos dentistas com os serviços, principalmente no que se refere a falta de regulação nos contratos de trabalho. A imparcialidade do Estado frente às decisões municipais deve ser revista; não no sentido retrógrado e arbitrário da gestão centralizada e inflexível, mas sim na busca da corresponsabilidade e transparência. Em 10 anos de Brasil Sorridente foram investidos mais de US\$2,6 bilhões na saúde bucal, um gasto necessário e com diversos benefícios para à população ${ }^{13}$. Nesse momento é necessário analisar os indicadores do impacto dessa e de outras políticas e ajustar os próximos passos com vistas à intersetorialidade das ações e no compromisso da redução das desigualdades sociais. Os países que planejam qualificar a força de trabalho em saúde, visando alcançar a cobertura universal, devem propor medidas de regulamentação em diferentes contextos, na formação, na gestão dos profissionais de saúde em todos os níveis do sistema e também a regulamentação do quadro de recursos humanos para a saúde, que deve ser orientada pelas necessidades e expectativas da população, levando em consideração a diversidade socioeconômica e cultural. Portanto, é necessário, entre outras medidas, o planejamento de estratégias voltadas aos RHS.

Os desafios impostos ao Brasil para superar as desigualdades em saúde e melhorar o provimento, impõem que a expansão dos postos de trabalho em odontologia continue, de modo a fortalecer a rede e permitir a integralidade do cuidado no SUS, entretanto, a continuidade dos êxitos alcançados demanda que medidas regulatórias dos contratos de trabalho e apoio aos gestores locais entrem na agenda das ações prioritárias da política Brasil Sorridente.

\section{Colaboradores}

M Gabriel: concepção do estudo, metodologia, recolha e análise dos dados, estruturação e escrita do artigo, e revisão da redação em todas as versões incluindo a final. MH Cayetano e MM Chagas: recolha e análise dos dados, estruturação e escrita do artigo em todas as versões, e revisão da redação final. ME Araujo, G Dussault, GA Pucca Junior e FCS Almeida: desenho do estudo e revisão da redação do artigo em todas as versões incluindo a final. 


\section{Referências}

1. Buchan J, Couper ID, Tangcharoensathien V, Thepannya K, Jaskiewicz W, Perfilieva G, Dolea C. Early implementation of WHO recommendations for the retention of health workers in remote and rural areas. Bull World Health Organ 2013; 91(11):834-840

2. Campbell J, Buchan J, Cometto G, David B, Dussault G, Fogstad H, Fronteira I, Lozano R, Nyonator F, Pablos-Méndez A, Quain EE, Starrs A, Tangcharoensathien V. Human resources for health and universal health coverage: fostering equity and effective coverage. Bull World Health Organ 2013; 91(11):853-863.

3. Dussault G, Dubois CA. Human resources for health policies: a critical component in health policies. Hum Resour Health 2003; 1(1):1.

4. World Health Organization (WHO), Global Health Workforce Alliance. Human resources for health: foundation for Universal Health Coverage and the post-2015 development agenda. Report of the Third Global Forum on Human Resources for Health. Genebra: WHO; 2013.

5. World Health Organization (WHO), Global Health Workforce Alliance. Dublin Declaration on Human Resources for Health. Building the health workforce of the future. Fourth Global Forum on Human Resources for Health; 2017 Nov 13-17; Dublin, Irlanda.

6. Oliveira FP, Vanni T, Pinto HA, Santos JTR, Figueiredo AM, Araújo SQ, Matos MFM, Cyrino EG. Mais Médicos: um programa brasileiro em uma perspectiva internacional. Interface (Botucatu) 2015; 19(54):623634.

7. Dussault G, Franceschini MC. Not enough there, too many here: understanding geographical imbalances in the distribution of the health workforce. Hum Resour Health 2006; 4:12.

8. Willis-Shattuck M, Bidwell P, Thomas S, Wyness L, Blaauw D, Ditlopo P. Motivation and retention of health workers in developing countries: a systematic review. BMC Heal Serv Res 2008; 8:247.

9. Kroezen M, Dussault G, Craveiro I, Dieleman M, Jansen C, Buchan J, Barriball L, Rafferty AM, Bremner J, Sermeus W. Recruitment and retention of health professionals across Europe: A literature review and multiple case study research. Health Policy 2015; 119(12):1517-1528.

10. Magnago C, Pierantoni CR, França T, Vieira SP, Miranda RG, Nascimento DN. Política de Gestão do Trabalho e Educação em Saúde: a experiência do ProgeSUS. Cien Saude Colet 2017; 22(5):1521-1530.

11. Martins MIC, Molinaro A. Reestruturação produtiva e seu impacto nas relações de trabalho nos serviços públicos de saúde no Brasil. Cien Saude Colet 2013; 18(6):1667-1676.

12. Petersen PE. Global policy for improvement of oral health in the 21st century--implications to oral health research of World Health Assembly 2007, World Health Organization. Community Dent Oral Epidemiol 2009; 37(1):1-8.

13. Pucca Junior GA, Gabriel M, Araujo ME, Almeida FC. Ten Years of a National Oral Health Policy in Brazil: Innovation, Boldness, and Numerous Challenges. $J$ Dent Res 2015; 94(10):1333-1337.

14. World Health Organization (WHO). Increasing Access to Health Workers in Remote and Rural Areas Through Improved Retention: Global Policy Recommendations. Genebra: WHO; 2010.
15. Brasil. Ministério da Saúde (MS). Departamento de Atenção Básica. Histórico de Cobertura da Saúde da Família [página na Internet]. [acessado 29 Dez 2017]. Disponível em: http://dab.saude.gov.br/portaldab/ historico_cobertura_sf.php

16. Brasil. Ministério da Saúde (MS). Banco de dados do Sistema Único de Saúde (DATASUS), Cadastro $\mathrm{Na}$ cional de Estabelecimentos de Atenção à Saúde (CNES) Recursos Humanos [página na Internet]. [acessado 29 Dez 2017]. Disponível em: http://tabnet.datasus.gov. $\mathrm{br} / \mathrm{cgi} / \mathrm{deftohtm}$.exe?cnes/cnv/prid02AC.def

17. Brasil. Ministério da Saúde (MS).e-Gestor atenção, informação e gestão da atenção básica [página na Internet]. [acessado 15 Jan 2018]. Disponível em: https:// egestorab.saude.gov.br/

18. Brasil. Ministério da Saúde (MS). Departamento de Atenção Básica. Programa de Melhoria do Acesso e da Qualidade dos Centros de Especialidades Odontológicas [página na Internet]. [acessado 16 Maio 2018]. Disponível em: http://dab.saude.gov.br/portaldab/ ape_pmaq.php?conteudo=1_ciclo_ceo

19. Gomes D, Ramos FRS. O profissional da odontologia pós-reestruturação produtiva: ética, mercado de trabalho e saúde bucal coletiva. Saude Soc 2015; 24(1):285-297.

20. Departamento Intersindical de Estatística e Estudos Socioeconômicos (DIEESE). Salário mínimo nominal e necessário [página na Internet]. [acessado $27 \mathrm{Jan}$ 2016]. Disponível em: http://www.dieese.org.br/analisecestabasica/salarioMinimo.html

21. Almeida G, Sarti FM, Ferreira FF, Diaz MD, Campino AC. Analysis of the evolution and determinants of income-related inequalities in the Brazilian health system, 1998-2008. Rev Panam Salud Publica 2013; 33(2):90-97.

22. Nogueira RP. O desenvolvimento federativo do sus e as novas modalidades institucionais de gerência das unidades assistenciais. In: Santos NR, Amarante PDC. Gestão Pública e Relação Público Privado na Saúde. Rio de Janeiro: Cebes; 2010. p. 24-47.

23. Girardi SN, Carvalho CL. Contratação e qualidade do emprego no programa de saúde da família no Brasil. In: Brasil. Ministério da Saúde (MS). Observatório de recursos humanos ou saúde no Brasil: estudos e análises. Rio de Janeiro: Fiocruz; 2003. p.157-190.

24. Faria HX, Dalbello-Araujo M. Precarização do trabalho e processo produtivo do cuidado. Mediações 2011; 16(1):142-156.

25. Brasil. Ministério da Saúde (MS). Secretaria de Gestão do Trabalho e da Educação na Saúde. Departamento de Gestão e da Regulação do Trabalho em Saúde. Programa Nacional de Desprecarização do Trabalho no SUS: Desprecariza SUS: perguntas \& respostas: Comitê Nacional Interinstitucional de Desprecarização do Trabalho no SUS/Ministério da Saúde, Secretaria de Gestão do Trabalho e da Educação na Saúde, Departamento de Gestão e da Regulação do Trabalho em Saúde. Brasília: MS; 2006.

26. Huicho L, Dieleman M, Campbell J, Codjia L, Balabanova D, Dussault G, Dolea C. Increasing access to health workers in underserved areas: A conceptual framework for measuring results. Bull World Health Organ 2010; 88(5):357-363. 
27. Chen L, Evans T, Anand S, Ivey Boufford J, Brown H, Chowdhury M, Cueto M, Dare L, Dussault G, Elzinga G, Fee E, Habte D, Hanvoravongchai P, Jacobs M, Kurowski C, Michael S, Pablos-Mendez A, Sewankambo N, Solimano G, Stilwell B, de Waal A, Wibulpolprasert S. Human resources for health: Overcoming the crisis. Lancet 2004; 364(9449): 1984-1990.

28. Brasil. Constituição da República Federativa do Brasil de 1988. Diário Oficial da União 1988; 5 out.

29. Conselho Federal de Odontologia (CFO). O que esperamos do próximo Presidente do Brasil: 2015-2108. Brasília: CFO; 2014.

30. Pan American Health Organization (PAHO), World Health Organization (WHO). Health in the Americas 2012 [Internet]. [acessado 22 Mar 2016]. Disponível em: http://www.paho.org/saludenlasamericas /index. php?option=com_docman\&task=doc_view \&gid $=118 \&$ Itemid $=$

31. Campos FE, Machado MH, Girardi SN. A fixação de profissionais de saúde em regiões de necessidades. $\mathrm{Di}$ vulg Saude Debate 2009; 44:13-24.

32. Brasil. Ministério da Saúde (MS). Diretrizes da política nacional de saúde bucal. Brasília: MS; 2004.

33. Brasil. Ministério da Saúde (MS). Passo a passo das ações do Brasil Sorridente [página na Internet]. [acessado 20 Mar 2016]. Disponível em: http://dab.saude. gov.br/portaldab/ape_brasil_sorridente.php

34. Roodenbeke E, Dussault G. Contracting health personnel. In: Perrot J, Roodenbeke E, editores. Strategic contracting for health systems and services. New York: Transaction Publishers; 2011. p. 249-284.

35. International Council of Nurses. International Hospital Federation. International Pharmaceutical Federation. World Confederation for Physical Therapy. World Dental Federation. World Medical Association. Guidelines: incentives for health professionals. Genebra: WHO; 2008

36. Cardoso AL, Stiebler V, Machado MH. Mercado de Trabalho dos Odontólogos no Brasil. Divulg Saude Debate 2010; 45:71-79.

37. Global Health Workforce Alliance (GHWA), World Health Organization (WHO). A universal truth: No health without a workforce. Third Global Forum on Human Resources for Health Report. Genebra: GHWA, WHO; 2013.
38. Medeiros CRG, Junqueira ÁGW, Schwingel G, Carreno I, Jungles LAP, Saldanha OMDFL. A rotatividade de enfermeiros e médicos: um impasse na implementação da Estratégia de Saúde da Família. Cien Saude Colet 2010; 15(Supl. 1):1521-1531.

39. Alves VS. Um modelo de educação em saúde para o Programa Saúde da Família: pela integralidade da atenção e reorientação do modelo assistencial. Interface (Botucatu) 2005; 9(16):39-52.

40. Mattos GCM, Ferreira EFE, Leite ICG, Greco RM. A inclusão da equipe de saúde bucal na Estratégia Saúde da Família: entraves, avanços e desafios. Cien Saude Colet 2014; 19(2):373-382.

41. Nogueira RP. Problemas de Gestão e Regulação do Trabalho no SUS. In: Nogueira RP, Piola SF, Vianna SM, Rodrigues VA. Tendência na evolução do emprego e nas relações de trabalho em saúde: a gestão de recursos humanos no Sistema Único de Saúde. Brasília: UNB; CEAM, NESP, ObservaRH; 2010. p.45-65.

42. Junqueira TDS, Cotta RMM, Gomes RC, Silveira SDFR, Siqueira-Batista R, Pinheiro TMM, Sampaio RF. As relações laborais no âmbito da municipalização da gestão em saúde e os dilemas da relação expansão/ precarização do trabalho no contexto do SUS. Cad Saude Publica 2010; 26(5):918-928.

43. Buchan J, Fronteira I, Dussault G. Continuity and change in human resources policies for health: lessons from Brazil. Hum Resour Health 2011; 9:17.

Artigo apresentado em 20/09/2017

Aprovado em 20/07/2018

Versão final apresentada em 22/07/2018 